\title{
Eine neue Ansicht über die Metrik des Romanos.
}

Zur Metrik des Romanos hat neuerdings Matthaios Paranikas eine Ansicht geäufsert, welche die bisherigen Ergebnisse von Mone, Pitra, Christ, Meyer und Krumbacher wieder in Frage stellt.

Nach den Untersuchungen dieser Gelehrten schien Folgendes festzustehen: In den byzantinischen Kirchenhymnen ist die antike quantitierende Poesie völlig verschwunden und durch die rhythmische Dichtung abgelöst worden. Nicht mehr giebt wie bei den Alten die Quantität der Silben den Ausschlag, denn die Silben haben jeden Unterschied der Quantität verloren. Wir haben es lediglich und allein mit einer accentuierenden Dichtung zu thun. Auch die alten Verse und Strophen wurden fallen gelassen. In der rhythmischen Hymnendichtung findet man keine Zusammenstellung antiker Fülse. Ihre Strophen bestehen aus meist kurzen, ungleichsilbigen Zeilen, die sich in jeder Strophe immer wieder an dem nämlichen Platze wiederholen. Durch den Mangel an Gleichzeiligkeit unterscheidet sie sich auch von den ebenfalls nach dem Accent gebauten politischen Versen. ${ }^{1}$ ) - Das war bis heute die Summe von der Metrik der byzantinischen Kirchenhymnen, deren schönste und beste wir unstreitig Romanos zu verdanken haben.

I.

Nun veröffentlicht Prof. Paranikas im Vizantijskij Vremennik 5

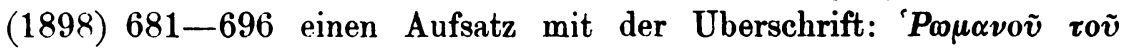

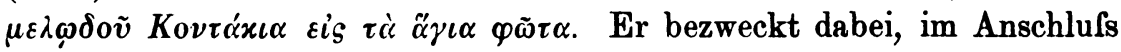
an dieses Lied auf Theophanie (= Epiphanie, 6. Januar) seine von der bisherigen Meinung abweichenden metrischen Ansichten darzulegen. Hierfür konstituiert er zuerst (S. 682-687) auf Grund der Ausgabe des Athosmönches Alexandros Eumorphopulos ${ }^{2}$ ) einen geeigneten Text. ${ }^{3}$ )

1) Vgl. Krumbacher, Byz. Litt.-Gesch.2 (1897) S. 690 f. u. dess. Studien zu Romanos (in den Sitzungsber. der philos.-philol. u. d. histor. Klasse d. k. bayer. Akad. d. W. 1898 Bd. II 69-268).

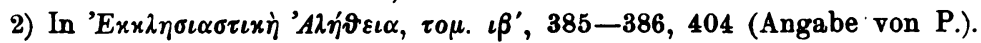

3) Die frühere von Pitra besorgte Ausgabe ist nicht berücksichtigt worden (vgl. Pitra, Analecta Sacra, tom. 1 (1876) p. 16 f.). 
„Weil nämlich“, schreibt er, „in einigen Versen, namentlich in der Mitte der Strophen, eine ziemliche Verwirrung herrscht, weil die Strophen teils zehn teils elf Verse haben und dadurch die Stichometrie in Unordnung gerät, so hielten wir es für gut, den Text auf zehnzeilige Strophen zurückzuführen, in denen sich dann die einzelnen Zeilen entsprechen, ferner unter Zuhilfenahme des Metrums an einigen Stellen Korrekturen anzubringen und so einen Text zu bieten, welcher nach unserer Ansicht richtiger ist." An die Darbietung des redigierten Textes schliefst sich (S. 687-689) eine knappe Inhaltsangabe des Gedichtes. Drittens endlich kommt (S. 689-695) die Hauptsache, die metrische Analyse.

Es ist nicht ganz leicht, den Gedankengang des Verfassers auf wenige klare Sätze zu bringen. Die zu erhärtende Behauptung finden wir mehr allgemein in den Worten auf S. 689, Abs. 4 ausgesprochen: „Die Byzantiner haben, ohne die Quantität oder das Zeitmafs zu vernachlässigen, in ihren Gedichten doch ganz besonders das accentuierende Metrum oder den Rhythmus zur Anwendung gebracht und eine derartige Mannigfaltigkeit in der künstlerischen Form der Verse, Absätze, Abschnitte und Strophen ihrer Lieder gezeitigt, dafs sie, wir können sagen, den Alten nicht viel nachstehen". Speziell von Romanos heifst es im folgenden Absatz: „Vor allem aber zeichnet sich hierin unser Romanos aus, welcher die alten metrischen Schemata auf der Basis des Rhythmus in reichlichem Mafse zur Anwendung gebracht hat".

Für letztere Behauptung wird sodann der Beweis versucht (S. 689695). Einstweilen nicht in dem Liede auf die heiligen Lichter selbst,

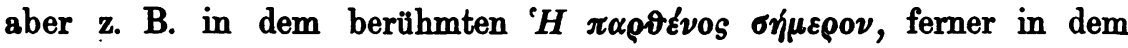

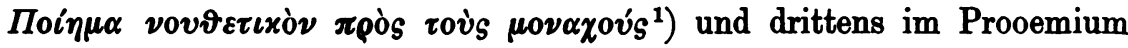

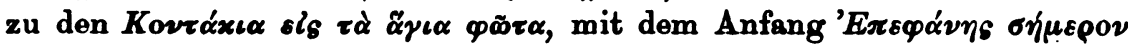

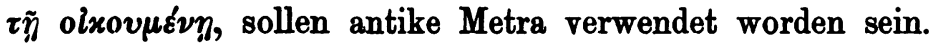

Das erstgenannte Prooemium gliedert P. in 6 Verse, die bis auf den letzten je eine oder zwei Abteilungen haben, so:

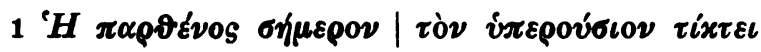

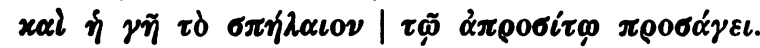

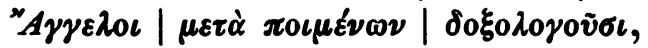

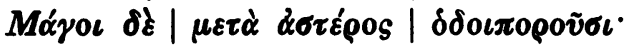

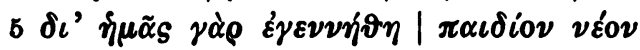

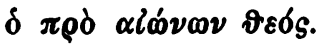

Der erste Vers, zu zwei Abteilungen, bestehe nun aus einem

1) Ed. Pitra, An. Sacr. I 44-52. P. benützt die Ausgabe in der 'Exxג.

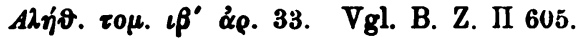


katalektischen trochäischen Dimeter und einem katalektischen daktylischen Trimeter. Also:

$$
-u-v|-u \geq \|-u v|-u v \mid-\underline{u} \text {. }
$$

Der zweite Vers desgleichen. Der dritte und vierte Vers, die wiederum einander parallel sind, haben je drei Abteilungen und bestehen aus 1) einem Daktylus, 2) einem hyperkatalektischen jambischen Monometer und 3) noch einmal einem solchen. Also:

$$
-v u\|v-v-|u \| v-v-| v \text {. }
$$

Man könne diese Verse aber auch ohne Abteilungen als katalektische daktylische Pentameter lesen, nämlich:

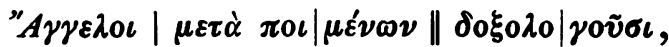

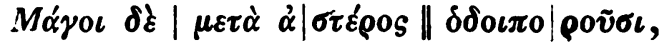

d. i.

$$
\text { -vu|-vv|--||-vu|-u. }
$$

Nur sei dabei, wie Figura zeigt, zu beachten, dafs den dritten Fufs ein Spondeus bilde.

Im fünften Vers, zu zwei Abteilungen, findet P. den zweimal gesetzten dritten Päon und einen hyperkatalektischen jambischen Monometer

$$
\text { vu-v|uv-uHu-v-|u. }
$$

Der sechste Vers endlich heifst ein hyperkatalektischer daktylischer Dimeter:

$$
-v u|-v u| v \text {. }
$$

Die beiden anderen Strophen brauche ich hier nicht vollständig anzuführen. Die eine, aus dem Gedichte an die Mönche, bestehe aus anapästischen Dimetern. Dabei hat der fünfte „anapästische“ Dimeter nach P. folgende merkwürdige Gestalt:

$$
u-v-1 u v-\therefore
$$

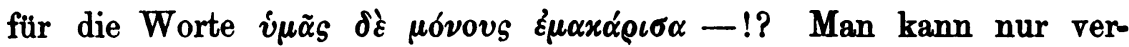
muten, dafs am Schlufs des ersten Metrums die Kürze übersehen wurde und dafs der letzte Anapäst durch einen Spondeus ersetzt sein soll. Keinenfalls ist es ein elfsilbiger Vers, wofür P. ihn ausgiebt $(,=\alpha \dot{\nu} \alpha \pi \alpha \iota \sigma \tau$.

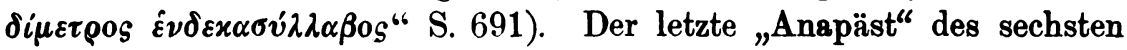
Verses wird so markiert: $\_\_$; der ganze Vers lautet:

das Schema dafür:

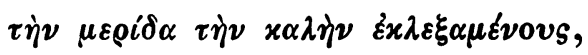

Das Prooemium zum Hymnus auf Theophanie endlich wird in vier Verse zerlegt. Die drei ersten seien rhythmische trochäische Trimeter:

$$
-v-v|-v-v|-v-v \text {, }
$$

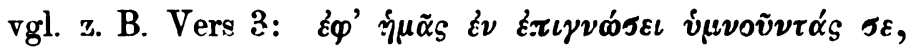
der letzte ein daktylischer Tetrameter:

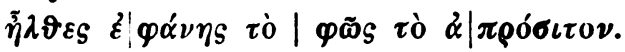


Dann fährt P. fort: „Und der Kehrvers dieser Strophe , $\eta \lambda \partial \varepsilon \varepsilon$

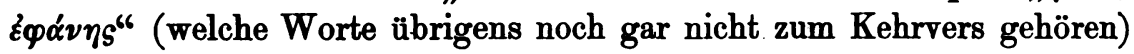
„führt uns nun zum Hauptthema, zur Analyse des oben wiedergegebenen Romanos-Hymnus auf die heiligen Lichter".

Folgendes ist die erste Strophe in der Gestalt, welche nach P.' Ansicht die richtigere ist:

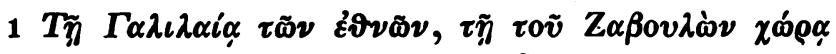

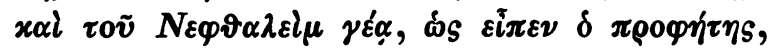

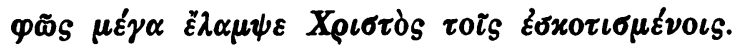

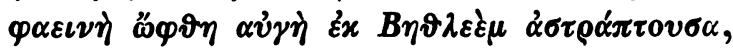

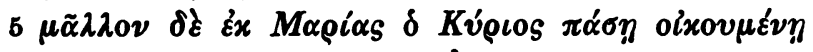

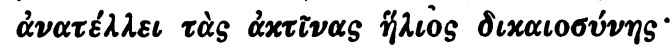

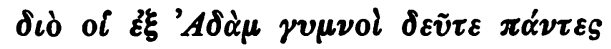

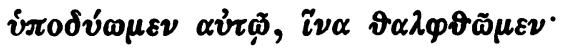

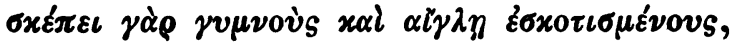

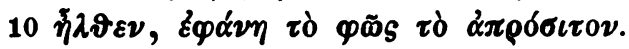

P. findet hier den eigentlichen politischen Vers einmal in seiner reinen Gestalt, die anderen Male in allerlei Verkürzungen oder Verlängerungen zur Anwendung gebracht. In seiner Textgestaltung ist allerdings der erste Vers jeder Strophe ein politischer Vers, nach der antiken Ausdrucksweise also ein fünfzehnsilbiger katalektischer jambischer Tetrameter, z. B. Vers 1:

$$
u-v-1 v-v-\| v-v-1 v-v
$$

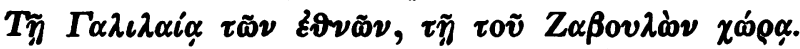

Im zweiten Vers zählen wir nur 14 Silben. Romanos habe die letzte Silbe des ersten Halbverses fallen gelassen und dadurch zwei ganz gleiche Halbverse erzielt, z. B. Vers 2:

$u-v-|v-u \| v-v-| v-v$

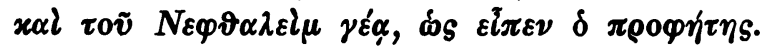

Im dritten Vers sei die letzte Silbe des zweiten Halbverses weggeblieben Der vierte Vers sei zur Abwechselung anstatt eines jambischen ein trochäischer Tetrameter, „das Gegenstück zum politischen Vers". Das erste Hemistichion des fünften Verses bestehe aus vier Daktylen, von denen aber entweder der erste oder der zweite Fufs durch einen Spondeus abgelöst werde; das zweite Hemistichion habe drei Trochäen. Der sechste Vers ist gar ein Sechzehnsilber, ein akatalektischer trochäischer Tetrameter. Der siebente Vers zerfällt in drei Jamben und zwei Anapäste „mit Katalexis“ so:

$$
u-v-|v-\|--| v v-1 v
$$

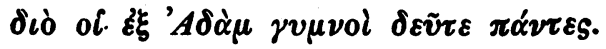


Der erste Anapäst wird aber offenbar durch einen Spondeus ersetzt. Im achten Vers findet P. einen katalektischen trochäischen Dimeter und dazu „zwei Daktylen, von denen der zweite katalektisch oder ein Trochäus ist". Der neunte Vers besteht aus einem katalektischen trochäischen Monometer und ferner drei Anapästen mit Katalexis, „von denen der erste dreisilbig und auf der zweiten Silbe betont ist (was bei den Byzantinern und bei Romanos selbst und im Akathistos gewöhnlich $)^{1}$ ); die beiden anderen sind $\mathrm{zweisilbig,} \mathrm{ihnen} \mathrm{folgt} \mathrm{eine}$ Silbe als Katalexis, und so entsteht der Paroemiacus der Alten" also:

$$
(-v-v \mid-\|) v-v 1--1--1 v
$$

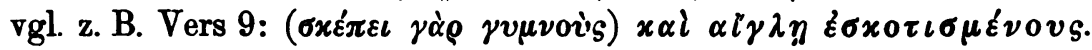
Der zehnte und letzte Vers der Strophe hat vier Daktylen.

„Und das ist", schliefst P. seinen Aufsatz, „die metrische Analyse des Gedichtes von Romanos. Was für Schlufsfolgerungen ziehen wir nun aus derselben?"

"Wir folgern, dals Romanos in den oben analysierten Gedichten fast alle Metra der Alten auf rhythmischer Basis zur Anwendung gebracht hat, einfache und gemischte; dafs bei ihm, soviel wir sehen, zum ersten Male der fünfzehnsilbige sogenannte politische Vers erscheint, der auch in der Kirche gebräuchlich ist und bis heute gesungen wird, wie wir anderswo aus anderen Gründen gezeigt haben; dals er aus diesem politischen Vers noch zwei weitere Schemata desselben abgeleitet hat, nämlich durch Streichung einer Silbe am Ende des ersten oder am Anfang des zweiten Hemistichion; dafs er nach dem Muster dieses jambischen politischen Verses einen ebensolchen fünfzehnsilbigen trochäischen gebildet hat; dafs er Trochäen und einfache Anapäste gebraucht hat, in mannigfacher Weise Daktylen mit Trochäen, Jamben mit Anapästen vermischt und so vielerlei rhythmische Schemata von Metren vorgeführt hat, woraus erwiesen wird, dafs die Byzantiner im Besitze der alten Bildung gewesen sind, dafs aber unser Romanos von den Neueren mit Recht der Pindar der christlichen Poesie genannt wird."

Ich glaube nichts Wesentliches von den Behauptungen und Beweisen des Verfassers äbergangen zu haben. Sehen wir nun, ob er im Rechte ist.

II.

Bei Untersuchungen wie die vorliegende ist es jedenfalls gut, möglichst voraussetzungslos an den überlieferten Text heranzutreten. Die Art und Weise der Überlieferung wird zuerst zu berücksichtigen sein. Bei Gedichten, die wie das Lied auf Epiphanie aus eigens dazu ge-

1) Von P. eingeklammert. 
schriebenen Gesangbüchern während des Gottesdienstes vorgetragen wurden, dürften etwaige Abteilungen von Wortgruppen oder die Andeutung von Sinnes- oder Sanges-Pausen, wenn solche sich finden, keinenfalls unbeachtet bleiben. Sollte man aber Grund zu der Vermutung haben, dafs bestimmte Versmalse und Strophenbilder zur Anwendung gekommen seien, so mürsten diese so viel als möglich aus dem Texte herausgelesen, nicht in denselben hineingetragen werden. Viel weniger noch geht es an, den überlieferten Text einzig und allein zu Gunsten eines im voraus ganz oder teilweise angenommenen Metrums zurechtzuschneiden. Ist der Text mehrfach überliefert, so müssen natürlich die verschiedenen Überlieferungen zum Vergleich herangezogen werden.

Es war deshalb von P. nicht wohlgethan, sich auf die Überlieferung jener Laura-Hs zu beschränken und die Ausgabe von Pitra, die auf wenigstens vier weiteren Hss fufst, aufser acht zu lassen. Abgesehen von allem andern hätten dann nicht Lücken der Laura-Hs durch blofse Konjekturen ergänzt zu werden brauchen, wie z. B. durch die äufserst merkwürdige Konjektur in V.164-166 bei P. = Strophe 16

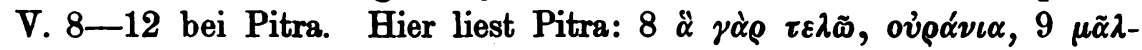

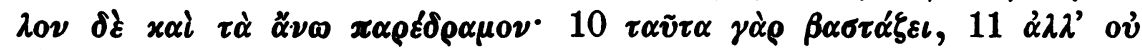

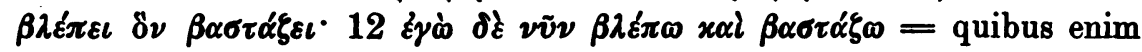
fungor, coelestia sunt, immo et superna transilivi: ea quippe ferunt, non vero cernunt quem sustinent; ego vero nunc et video et fero. (Es spricht Johannes, der eben den Heiland tauft und aus dem Jordan hebt.) $\mathrm{Da}$ in der Laura-Hs zwischen V. 10 und V. 12 nach Pitras

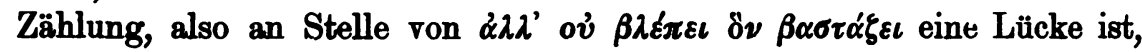
ergänzt nun $P$., wegen seines Metrums zugleich unter Streichung zweier

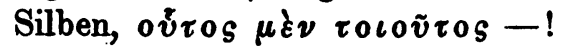

Aber bedeutend sohlimmer ist die eigenmächtige Verarbeitung des überlieferten Textes. Wenn keine einzige Strophe unverändert bleibt, wenn in den meisten Strophen zweimal, ja dreimal eine oder gar

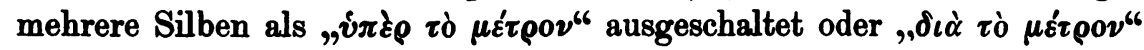
ergänzt werden, kann man sich eines starken Vorurteils gegen dieses unduldsame Metrum bereits kaum mehr erwehren. So verderbt pflegen die liturgischen Hss der Byzantiner nun doch nicht leicht zu sein, und ich kann versichern, dafs sie es in unserm Falle auch in der That nicht sind. Weil, wie jedermann weils, Pitras Apparat bisweilen trügt, so hat mir mein verehrter Lehrer, Herr Prof. Krumbacher, auf meine Bitte in freundlichster Weise die Einsicht in sein handschriftliches Material gestattet. Dort habe ich von einer ,ziemlichen Verwirrung, namentlich in der Mitte der Strophen" nichts 'gefunden. Ich fand bestätigt, was ich schon aus Pitra vermutete, dafs P. bei seinen Korrek- 
turen fast durchweg die klare Überlieferung sämtlic̣her Hss gegen sich hat. ${ }^{1}$ )

Indes müssen wir auf die metrische Analyse und die Beweisführung daraus etwas näher eingehen. Zwei Hauptfragen kommen dabei in Betracht, die P. zwar nicht so klar geschieden hat, die ich aber zur Erleichterung der Ubbersicht im Folgenden auseinanderhalten will: 1) Hat Romanos sich der antiken Metra bedient? 2) Hat er den politischen Vers angewandt, welcher in der späteren mittelund neugriechischen Poesie eine so grofse Rolle spielt? P. antwortet auf beide Fragen mit einem begeisterten Ja.

1) Für die antiken Metren argumentiert $P$., wie wir sahen, zu-

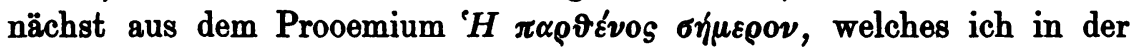
von ihm beliebten Form abgedruckt habe. Mufs es nicht auffallen, dals dabei von fünf Versen sich drei je einem, die beiden übrigen sogar je zwei Einschnitten unterwerfen müssen, um der Ehre antiker Titel würdig zu werden? Und wenn jeder Vers nur wenigstens einen einzigen, einheitlichen Namen bekäme! Der dritte und vierte Vers erhalten dreigespaltene Überschriften, wenn sie nicht vorziehen, ohne den zweifachen Schnitt als daktylische Pentameter aufzutreten, was jedoch gleichfalls seinen Haken hat.

Würde es sich übrigens um die klassischen Namen allein handeln, so brauchte man schliefslich nicht viel Wesens daraus zu machen. Die Metrik der Alten hat eine so reiche Auswahl von Termini für alle erdenklichen Fufsarten, dafs wir mühelos auch für die bisher anerkannten kurzen Verse in den Romanos-Hymnen klassische Bezeichnungen anwenden könnten. Aber jedenfalls mufs festgehalten werden, dafs es sich lediglich und allein um Accentpoesie und nirgends mehr um Quantitätspoesie handelt. Wenn man also bei der rhythmischen Dichtung, wie es ja auch vielfach bei modernen Gedichten geschieht, den Rhythmus durch die antiken Zeichen und Namen andeuten will, so darf man das Zeichen für eine Länge in der alten Quantitätsdichtung jetzt

1) Es sind Patmiacus 212 saec. 11, Corsinianus 366 saec. 11, Mosquensis Synod. 437 saec. 12, Taurinensis B. IV 34 saec. 11 und für die späteren Strophen auch Vindobonensis suppl. graec. 96 saec. 12. - Vgl. z. B. die drei ersten Strophen:

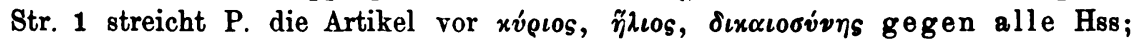

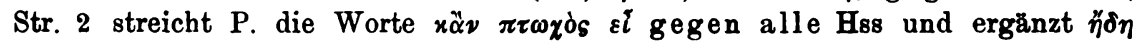
gegen alle Hss; Str. 3 streicht $P$. zweimal das $\varepsilon \nu$, vor $\tilde{v} \delta \alpha \sigma \iota$ und vor $\delta \iota \alpha \nu o i \alpha \iota$, gegen alle Hss. Die von $P$. so eigentümlich ersetzten Worte $\alpha \lambda \lambda^{\prime}$ ov $\beta \lambda \dot{\varepsilon} \pi \varepsilon \iota$, ô $\nu \alpha \alpha \sigma \alpha \dot{\xi} \xi \iota$ (s. oben), finden sich in allen Hss. Auch seine Korrektur von $\beta \alpha \sigma \tau \alpha \dot{\xi} \omega \omega$

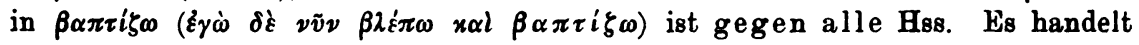
sich hier um die von Romanos so sehr beliebte Form der Antithese; vgl. über diese Krumbacher, Studien zu Romanos S. 205, 211, 215 ff., 264. 
nur für accentujerte Silben gebrauchen. Die übrigen Silben müssen als unbetont gelten, und nur unter dieser Bedingung und auf dieser Grundlage kann dann zur Namengebung für die Verse und Versglieder geschritten werden. In Bezug auf das Griechische haben wir Deutsche hier den kleinen Vorteil, dafs wir von Jugend auf die klassische griechische Poesie auch wirklich nach der Quantität lesen, selbst mit Vernachlässigung des Wortaccentes. Das Neugriechische hingegen, welches ebenso wie die anderen modernen Sprachen bezüglich der Quantität indifferent und einzig dem Gesetze der Betonung unterworfen ist, lesen wir auch nach der Betonung. Einem geborenen Griechen, der Altes und Neues auf dieselbe Weise liest, mag die Unterscheidung schwieriger sein. Es scheint, als ob. P. von dieser Schwierigkeit beeinflufst worden wäre. So z. B. in V. 2 und 3 von 'H $\pi \alpha \rho \vartheta \varepsilon^{\prime} v o s$, die er als daktylische Pentameter bezeichnet, in denen der dritte Daktylus durch einen Spondeus ersetzt wurde. Aber die letzten Silben von $\pi 0 \iota \mu \varepsilon ́ v \omega \nu$ und

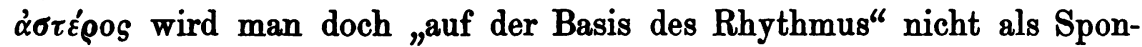
deen hinnehmen. In dem Gedichte an die Mönche scheinen Spondeen für Anapäste eintreten zu müssen; denn von den Schlufsworten der

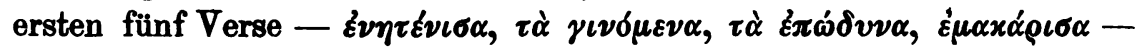
werden jedesmal die drei ersten Silben als Anapäst ( $u_{-}$) notiert, für die beiden folgenden steht wenigstens im ersten und fünften Verse ausdrücklich ein Spondeus (_ _). Dafs dies die Meinung von P. ist, mufs man auch daraus folgern, dafs er die Verse mit keinem Worte als katalektische bezeichnet, was er sonst, wo nötig, nie zu thun unterläfst. Im Hymnus auf die heiligen Lichter müssen Silben wie 'Io@ von

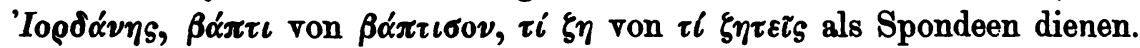
Auf das merkwürdige zweite Hemistichion im neunten Verse dieses Hymnus habe ich schon hingewiesen. Das Schema war: $v-v|--|--\mid$, der Name: „Drei Anapäste, von denen der erste dreisilbig und auf der zweiten Silbe betont, die beiden anderen zweisilbig (also Spondeen) ${ }^{1}$ ) sind; ihnen folgt noch eine Silbe als Katalexis". Indes treffen hier keineswegs zwei betonte Silben auf einander. Ein Spondeus ist also nicht vorhanden.

Aber kann denn bei der Accentpoesie von einem Eintreten des Spondeus für Daktylus und Anapäst überhaupt auch nur. die Rede sein? Kann eine Länge zwei Kürzen ersetzen, wenn es einen Unterschied zwischen Länge und Kürze überhaupt nicht mehr giebt? Das Übersehen dieses Punktes scheint mir eine grofse Schuld an P?' Irrtum zu tragen.

1) natürlich von mir eingefügt. 
Dazu mufs sich der Accent, mag er nun wollen oder nicht, ziemlich oft einfach in das Versmafs ergeben, z. B. wenn der Vers $\tau \dot{\eta} \nu$

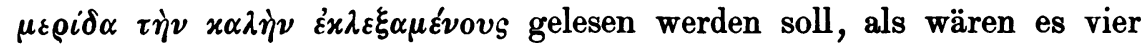

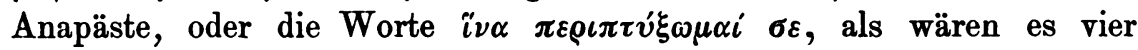
Jamben. Für den "christlichen Pindar" sind diese Leistungen doch recht mittelmälsig. Man freut sich um so mehr im Besitze der durch die Überlieferung bezeugten, schön in Absätze und Abschnitte gegliederten Strophen mit den kurzen, rhythmischen Versen, in die sich jedes Wort ganz zwanglos fügt.

2) Über das Alter des politischen Verses der Byzantiner hat $P$. auch in den Nachrichten (Izvjestija) des russ. archäol. Instituts in Kpel 2 (1897) 185-190 gehandelt. Er schliefst sich dort zunächst an Krumbachers Litteraturgeschichte (2. Aufl. S. 650-652) an. Danach hat Konstantin Porphyrogennetos, der in der ersten Hälfte des 10. Jahrhunderts schrieb, einige politische Verse in einer volksmälsigen Akklamation überliefert. Bedeutend älter sind die politischen Verse, die Johannes Klimax (c. 525-c. 600) und Johannes Moschos $(\dagger 629)$ in mehreren Sprichwörtern anführen. Man kann zum mindesten die bei Klimax getrost bis ins 5. Jahrhundert zurückdatieren, weil ja derartige Sentenzen eine geraume Zeit brauchen, bevor sie als Sprichwörter in den Volksmund übergehen. Aber es sind doch immer nur vereinzelte Verse, die sich so finden. $\mathrm{Zu}$ grölseren Gruppen vereint tritt der jambische Fünfzehnsilber vor dem 10. Jahrhundert nicht auf.

P. weist nun in dem obigen Aufsatze ein neues Beispiel dieses Verses und zwar bei Romanos nach. Es ist eben die erste Strophenzeile des Liedes auf die heiligen Lichter, welchem er dann im J. 1898 noch einmal die hier besprochene Untersuchung im Vizantijskij Vremennik gewidmet hat. In der That, wenn man das Lied so schreibt, wie $P$. es thut, kann die erste Zeile als politischer Vers gelesen wèrden. Und das bleibt auch bestehen, wenn wir andern es vorziehen, diese erste Zeile in zwei selbständige Verse zu zerlegen. Dann machen eben diese zusammen einen politischen Fünfzehnsilbler aus. Es ist P.' Verdienst, darauf hingewiesen zu haben. Die beiden Hälften jenes Verses, die eine zu acht, die andere zu sieben Silben, passen übrigens ganz wohl in das Gefüge der Hymnenstrophe. Dafs sie zudem leicht zu melodisieren sind, zeigt mehr als eines unserer deutschen Volkslieder. ${ }^{1}$ ) So wäre es also fast zu verwundern gewesen, wenn dieselben nicht auch einmal in einem der vielen Lieder des bedeutendsten byzantinischen Meloden vorkämen, zumal, wie wir oben sahen, einzelne solche Verse

1) Z. B. das bekannte „O Tannenbaum, o Tannenbaum". 
schon damals im Volksmunde lebten. Aber von Romanos nun gleich eine ganze Strophe, einen ganzen Hymnus auf dieses Schema aufgebaut sein lassen, scheint doch ein gewagtes Unternehmen. Das mülste schon auf kräftigere Beweise gestützt werden, als es $P$. gethan hat.

Erinnern wir uns an seine oben skizzierte Analyse der zehn Verse. Wenn wir bei der zweiten und dritten Zeile noch eine Nachwirkung des politischen Verses zugeben könnten, so haben doch die folgenden Zeilen mit ihren Trochäen, Daktylen, Spondeen und Anapästen aufser der grofsen Länge gewifs gar nichts, was an den politischen Vers als an ihre beabsichtigte Grundlage denken liefse. Und die Länge dieser Vierzehn-, Fünfzehn- und Sechzehnsilbler spricht von vorne herein und entschieden gegen die ganze Aufstellung überhaupt. Man braucht auch nur darauf zu achten, wie $P$. hier wieder den meisten seiner Verse zwiefachen Namen geben mufs, um einzusehen, dafs der Dichter selbst schwerlich diese abgeschlossenen, oft ganz verschiedenartigen Stücke als Teile von Ganzrersen aufgefalst hat noch aufgefalst wissen wollte. Gegen die zehnte Zeile könnte noch bemerkt werden, dafs der Refrain einen eigenen Vers zu bilden pflege. Refrain sind aber hier nur die

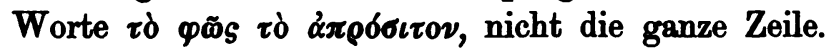

Nach dem Gesagten darf man die Schlufsfolgerungen, die P. aus seiner metrischen Analyse zieht, zum mindesten als sehr überschwenglich bezeichnen. Die erste derselben, dafs nämlich Romanos in einem einzigen Gedichte und noch drei Strophen fast alle Metra der Alten zur Anwendung gebracht habe, möchte ich überdies eher für einen Tadel als für ein Lob halten. Einzuräumen ist, dafs sich aus den beiden Gliedern der ersten Strophenzeile in P.' Textgebung ein politischer Vers machen läfst. $O b$ aber Romanos ihn als solchen erkannt oder gar beabsichtigt hat, fragt sich. Es ist wohl zu verneinen. Auch dürfte der Vers hier nicht zum ersten Male vorkommen. Die Sprichwörter, welche Johannes Klimax mitteilt, scheinen älter zu sein. Übrigens ist zu betonen, dals diese Sprichwörter ebenso wie jene Stücke des Lichterhymnus offenbar beide nichts mehr sind als erstmalige, unbewurste Anklänge oder Ansätze, aus denen sich erst allmählich und mit der Zeit der politische Vers entwickelt hat. Dafs ursprünglich seine beiden Teile selbständig vorgekommen sind, darf man auch ohne Rücksicht auf die thatsächlichen Belege daraus schliefsen, dafs die Cäsur nach der achten Silbe als ein unerläfsliches Erfordernis erscheint und dafs sie nach keiner anderen Silbe statthaben kann. - Sicher unbewiesen bleibt die Behauptung, der Dichter habe sozusagen die ganze übrige Strophe des analysierten Liedes aus immer neuen Variationen der ersten politischen Zeile aufgebaut. Das Schema des zweiten und dritten Verses 
bei P. erklärt sich viel wahrscheinlicher aus der eben erwähnten ursprünglichen Selbständigkeit der Hemistichien. Infolge derselben konnte sich das gleiche Hemistichion leicht mehrmals hintereinander wiederholen und sowohl Abstriche als auch Zusätze erfahren. Bezüglich der antiken Metra liefe die Sache auf einen blofsen Wortstreit hinaus, wenn nicht $P$. den Accenten so häufig Gewalt anthäte. Dagegen ist Einsprache zu erheben. Pindar der christlichen Poesie wurde Romanos von den Neueren genannt wegen der Verwandtschaft mit jenem Klassiker in der dithyrambischen Form und im erhabenen Schwung der Gedanken, nicht deshalb, weil er antike Versfüfse gebraucht hat.

Endlich wird auch P. zugeben nüssen, dafs eine Frage von dieser Bedeutung nicht auf Grund der Analyse eines einzelnen Hymnus gelöst wird. Es mü fsten viele Hymnen zum Vergleiche herbeigezogen werden, wie das bei seinen Vorgängern geschieht.

Valkenburg (L.) Holland.

Konrad Kirch, S. I. 